\title{
Environmental Factors Affecting Rose Downy Mildew and Development of a Forecasting Model for a Nursery Production System
}

\author{
B. J. Aegerter, Department of Plant Pathology, University of California, Davis 95616, J. J. Nuñez, Cooperative \\ Extension, Kern County, Bakersfield, CA 93307, and R. M. Davis, Department of Plant Pathology, University of \\ California, Davis 95616
}

\begin{abstract}
Aegerter, B. J., Nuñez, J. J., and Davis, R. M. 2003. Environmental factors affecting rose downy mildew and development of a forecasting model for a nursery production system. Plant Dis. 87:732-738.

The effect of various environmental parameters on rose downy mildew caused by Peronospora sparsa was determined under controlled conditions and in the field. In growth chambers, optimal temperatures for infection and colonization of rose leaves were 15 to $20^{\circ} \mathrm{C}$ and 20 to $25^{\circ} \mathrm{C}$, respectively. At optimal temperatures, infection required only $2 \mathrm{~h}$ of leaf wetness, although disease severity increased significantly with an increasing duration of leaf wetness up to $10 \mathrm{~h}$. Infection of leaves occurred at temperatures as low as $5^{\circ} \mathrm{C}$ with $8 \mathrm{~h}$ of leaf wetness. The latent period of infection varied from 4 to 7 days. Weather and disease incidence data collected from natural field epidemics were used in the development of a predictive model of rose downy mildew. Logistic regression was used to identify those weather variables that explained the largest portion of the variation in disease incidence. The optimum regression model incorporated three weather variables calculated as cumulative totals over the previous 10 days: (i) hours of leaf wetness when temperatures were less than $20^{\circ} \mathrm{C}$ (positive correlation); (ii) hours between 15 and $20^{\circ} \mathrm{C}$ (negative correlation); and (iii) hours when temperatures exceeded $30^{\circ} \mathrm{C}$ (negative correlation). The simplest model, which was also a good fit, included only the 10-day cumulative number of hours of leaf wetness. The critical number of hours of leaf wetness for disease development was an average of $8.4 \mathrm{~h}$ per day over 10 days.
\end{abstract}

More than $50 \%$ of the bare-root roses produced in the United States are grown near Wasco, in the San Joaquin Valley, California. In 2000, over 23 million rose plants were harvested at a farm-gate value of $\$ 46$ million (11). Since the early 1990s, this nursery production system has had a sporadic but continuing problem with rose downy mildew, caused by the oomycete Peronospora sparsa. Infected leaves quickly abscise, often resulting in severe defoliation (1) and reduced plant vigor. During the first 2 months after budbreak, rootstock cuttings that are defoliated by downy mildew may fail to root, resulting in reduced stands. On older bushes, the reduced vigor can result in failure of the canes to reach the diameter required for the top grade and such plants are often never harvested. Dieback of young canes has been observed when the stems are severely diseased, presumably due to invasion by secondary pathogens such as Botrytis spp.

Downy mildew is currently controlled with foliar fungicides that are applied preventatively. Although the disease occurs

Corresponding author: B. J. Aegerter

E-mail: bjaegerter@ucdavis.edu

Accepted for publication 31 January 2003.

Publication no. D-2003-0407-01R

(C) 2003 The American Phytopathological Society only sporadically, growers apply fungicides routinely because losses are potentially devastating. For these reasons and because downy mildew is apparently limited by some environmental conditions, it is a good candidate for forecasting (4). Also important for a successful prediction system is the current availability of chemical controls with some curative activity, such as metalaxyl. This allows the grower some latitude in timing applications once a disease warning has been issued. Disease warning or advisory systems have been developed to predict downy mildew occurrence in a number of other crops $(6,7,12,16)$. Systems such as these can reduce the number of fungicide sprays needed or improve disease control by optimizing the timing of application.

Very little is known about the role of environmental factors in the epidemiology of rose downy mildew. In greenhouse production systems, there is anecdotal evidence that some control of the disease can be achieved by temporarily raising the temperature to $27^{\circ} \mathrm{C}$ and ventilating the greenhouse to reduce humidity (1). Controlled studies of Peronospora sparsa conducted under laboratory conditions quite unlike those in the field have yielded varying results $(1,3)$. The variation between isolates studied by Breese et al. (3) indicates that isolates from different hosts and/or geographic locations may vary in their response to temperature.
The objectives of this work were to (i) investigate the effects of key environmental parameters on infection, colonization, and sporulation by California isolates of $P$. sparsa under controlled conditions; and (ii) use the information thus obtained in conjunction with data collected from natural field epidemics in the development of a predictive model of disease outbreaks.

\section{MATERIALS AND METHODS}

Experiments in controlled environments. Detached leaves used in controlled experiments were harvested from roses (cv. Europeana and numbered seedling 9102522) grown in the greenhouse in 5gallon pots. Greenhouse temperatures ranged from a minimum of $20^{\circ} \mathrm{C}$ to a maximum of 27 (winter) or $33^{\circ} \mathrm{C}$ (summer). Since replicated inoculations were spread over the course of a year, the test leaves grew under a variety of light, day length, humidity, and temperature conditions. Only recently expanded, succulent leaves were harvested; these were typically located above the sixth leaf node down from the shoot apex. Plants were maintained free of powdery mildew by sulfurburners placed in the greenhouses. Plants were fertilized weekly by drenching with full-strength Hoagland's solution.

Isolates of $P$. sparsa were originally collected from symptomatic leaves from nursery rose fields in Kern County, CA. To induce sporulation, leaves were incubated overnight at room temperature and high relative humidity $(100 \%)$. The leaves were washed with distilled water and the washate was filtered with a $12-\mu \mathrm{m}$ filter membrane (Isopore filter, Millipore Corp., Bedford, MA) to concentrate spores and remove any leaf surface chemicals. Spores were washed off the membrane filter with a solution of $0.1 \%$ Tween 20 and adjusted to approximately 40,000 sporangia per $\mathrm{ml}$ using a hemacytometer. This spore suspension was applied to the detached leaves with an airbrush sprayer until they were fully wetted. Inoculated leaves were incubated in darkness overnight on wire racks overlying water in plastic boxes $(24 \times 32 \times$ $11 \mathrm{~cm})$. After overnight incubation, leaves were allowed to dry to remove surface water. Inoculated leaves were then incubated at room temperature $\left(22^{\circ} \mathrm{C}\right)$ with alternating light/dark cycles supplied by a combination of wide-spectrum and coolwhite fluorescent lights. Spores were trans- 
ferred to fresh leaves after 7 to 10 days. In this way, field isolates were maintained in the laboratory for future experiments.

For studies on the effect of leaf wetness duration and temperature on infection, petioles of detached leaves were placed in distilled water in 1.5-ml microcentrifuge tubes. The tubes were placed on racks that were placed in plastic boxes over water to maintain high relative humidity. The boxes were kept in incubators at their respective test temperature overnight prior to inoculation in the morning. The leaves were inoculated as described above and each set was immediately returned to its respective test temperature. At various intervals after inoculation $(2,4,6,8,10,12$, and $24 \mathrm{~h})$, three leaves were removed from the box and placed in front of a small fan to evaporate the free water on the leaves. The drying time was typically 10 to $15 \mathrm{~min}$. Once dry, the leaves were returned to the incubator, but this time to a plastic box with relative humidity $(\mathrm{RH})$ below saturation. $\mathrm{RH}$ levels were maintained between 90 and $100 \%$ by salt solutions lining the bottom of the boxes. The following saturated salt solutions were used: potassium chloride (at 5 and $10^{\circ} \mathrm{C}$ ); potassium nitrate (at $15^{\circ} \mathrm{C}$ ); and magnesium sulfate (at 20 and $25^{\circ} \mathrm{C}$ ) (8). Humidities were measured with small data-logging RH/temperature sensors (HOBO H8 Pro, Onset Computer Corp., Pocasset, MA). Although the humidity levels fluctuated due to opening the box and the transpiration of the leaves, free water was observed only occasionally on the inside surface of the box lid.

Twenty-four hours after inoculation, boxes were removed from the test temperatures and were subsequently incubated in the lab at room temperature $\left(22^{\circ} \mathrm{C}\right)$ with alternating light/dark cycles provided by a combination of full-spectrum and coolwhite fluorescent lights. Five days after inoculation, the leaves were rated using a subjective scale: $0=0$ to $1.2 \% ; 1=1.3$ to $6.2 \% ; 2=6.3$ to $15.5 \% ; 3=15.6$ to $28 \% ; 4$ $=28.1$ to $42.5 \% ; 5=42.6$ to $57.5 \% ; 6=$ 57.6 to $72 \% ; 7=72.1$ to $84.5 \% ; 8=84.6$ to $93.7 \% ; 9=93.8$ to $98.7 \%$; and $10=98.8$ to $100 \%$ of the surface area of the leaves exhibiting symptoms of downy mildew. There were three leaves per treatment combination and replicate inoculations were conducted over time; inoculation of the $\mathrm{cv}$. Europeana was replicated four times and inoculation of the numbered seedling 91-02522 was replicated four times. All inoculations were conducted with Kern County isolate 99-1.

Another set of experiments was conducted to determine the effect of incubation temperature on the length of the latent period (time from infection to sporulation) and incubation period (time from infection to symptom appearance). For these studies, leaves were prepared and inoculated as described above for infection experiments. After inoculation, the leaves were incu- bated overnight (12 to $14 \mathrm{~h}$ ) in the dark at room temperature and $100 \% \mathrm{RH}$. In the morning, any remaining free water was evaporated with small fans, and the boxes were transferred to growth chambers (Conviron Model E15, Controlled Environments Limited, Winnipeg, Manitoba, Canada) at test temperatures of 5, 10, 15, 20 , and $25^{\circ} \mathrm{C}$. The growth chambers supplied $12 \mathrm{~h}$ of light consisting of a combination of metal halide and incandescent bulbs. The temperature of each chamber was automatically adjusted during the light period to compensate for the slight greenhouse effect within the boxes. The temperature inside the boxes was monitored and recorded with a small, data-logging temperature sensor (HOBO H8 Pro, Onset Computer Corp.). Beginning $72 \mathrm{~h}$ after inoculation, the proportion of leaves that were symptomatic and the proportion that were sporulating were recorded daily until all leaves were symptomatic and sporulating, or until 10 days after inoculation, whichever occurred first. There were 20 leaves per treatment. Replicate inoculations were conducted over time; inoculation with isolate 99-1 was replicated six times while inoculation with isolate 99-2 was replicated 3 times. These isolates were collected from two fields in Kern County in 1999.

Data analysis. For analysis of variation in infection levels, symptoms, and sporulation, the replicate inoculations were treated as blocks. Homogeneity of variance between blocks was tested to determine whether the infection level varied significantly among inoculations. The infection experiments were analyzed as a split-plot design, with temperature $(T)$ as the main plot and leaf wetness duration $(w)$ as the split-plot. For the colonization experiments, the variation in the proportion of diseased plants over time was analyzed. All analyses were done with the SAS statistical package (SAS Institute Inc., Cary, NC).

Response surface models of the effect of $T$ and $w$ on infection were developed using regression analysis. Various linear models (second-order polynomials of $T$ and $w$ with and without interaction terms) were evaluated by fitting linear equations to polynomial transformations of the data using the REG procedure in SAS. Additionally, nonlinear regression was used to develop a model based on a set of equations proposed by Duthie $(5,9)$ to describe the response of foliar pathogens to the combined effects of temperature and duration of wetness. The response to temperature was described by

$$
Y_{\max T}=f(T)=E /\left\{\exp \left[(T-F)^{2} G\right]\right\}
$$

where $Y_{\max T}$ is the maximum infection level at a given temperature, $E$ is the maximum when the temperature is optimal, $T$ is the temperature in degrees Celsius, $F$ is the optimal temperature, and $G$ is the intrinsic rate of decline from the maximum infection level as the tempera- ture deviates from optimal (equation 9 of Duthie, 9). To reduce the number of parameters to be estimated, $E$ was set to 10 . The parameters $F$ and $G$ were estimated from analysis of the maximum infection levels of each replicate at each temperature using the Marquardt iterative method of the NLIN procedure in SAS. In a second step of model development, the response of relative infection to the combined effects of temperature and wetness duration was described by

$$
Y=f(w, T)=f(T) \times\left\{1-\exp \left[-(B w)^{D}\right]\right\}
$$

in which $f(T)$ is calculated by the equation above, $B$ is the intrinsic rate of increase in infection level as duration of wetness increases, $D$ is the portion of the period of wetness in which the response decelerates, and $w$ is the wetness duration in hours (9). The parameter $C$ of Duthie (9) was set to zero based on the assumption that the infection process responds immediately to leaf wetness after a minimum duration of 2 h. Parameters $B$ and $D$ were estimated from the infection data from each replicate and each combination of temperature and duration of wetness using the Marquardt iterative method of the NLIN procedure.

For the nonlinear model, a pseudo- $R^{2}$ measure was obtained by calculating one minus the ratio of the sum of squares for residual to the corrected total sum of squares. Additionally, regression of observed against predicted infection levels was conducted and estimates of the intercept and slope parameters were evaluated.

Modeling of field epidemics. Field microclimate data were collected during the spring of 1998, 1999, and 2000. Weather stations (remote measuring station A730MD, Adcon Telemetry Inc., Boca Raton, FL) located within commercial rose nursery fields measured temperature, $\mathrm{RH}$, leaf wetness, and rainfall. A "combisensor" (Humitter 50YA, Vaisala Oyj, Helsinki, Finland) with a Pt100 temperature element and a Intercap RH sensor element (Vaisala Oyj) contained within plastic housing (to minimize solar and ground radiation effects while allowing air movement) was placed within the canopy. A resistance grid $(3 \times 4 \mathrm{~cm})$ located on the top of the temp$\mathrm{RH}$ sensor housing measured leaf wetness on a scale from 0 (high resistance, low wetness) to 10 (low resistance, high wetness). A value of three or greater was defined as wet. A datalogger in each weather station recorded the measurements for these variables each minute. Values averaged each 15 min were sent via radio signals to a receiver (A730SD, Adcon Telemetry Inc.) connected to a computer at a base station maintained by Western Farm Service (Fresno, CA). A computer at UC Davis downloaded the data from the base station computer daily via a modem.

In one location in 1998, a different type of weather station was used (CR10X Measurement and Calibration System, 
Campbell Scientific, Inc., Logan, UT), which recorded air temperature, $\mathrm{RH}$, and leaf wetness. A HMP35/45C temperature$\mathrm{RH}$ probe in a radiation shield (Model 41002, Campbell Scientific, Inc.) was placed within the canopy. A resistance grid (Model 237, Campbell Scientific, Inc.) located within the canopy measured leaf wetness on a scale from 6,999 (high resistance, low wetness) to 0 (low resistance, high wetness). A value of 28 or lower was defined as wet. Data were recorded once a minute and averaged each hour; these hourly averages were stored in the datalogger memory until they were downloaded weekly via a laptop computer.

Meteorological data were also retrieved from a weather station maintained by the State of California Department of Water Resources as part of the California Irrigation Management Information System (CIMIS) network. The station we accessed is located at the USDA Cotton Research Station just north of Shafter, CA, within a few kilometers of the rose production area.

To calculate the variables of interest for use in statistical analysis (i.e., number of hours of leaf wetness, average daily temperature, etc.) the data from the weather stations were imported into the computer program Subvariable 3.0 (Michael D. Cahn, UC Cooperative Extension, Monterey County). Candidate variables were included in logistic analysis based in part on the results from our studies in controlled environments described above. Hours at temperature intervals were calculated in intervals of $5^{\circ} \mathrm{C}$ within the range of 10 to $30^{\circ} \mathrm{C}$. Hours below $10^{\circ} \mathrm{C}$ and above $30^{\circ} \mathrm{C}$ were also calculated. Hours at relative humidity intervals were calculated from 65 to $95 \% \mathrm{RH}$ at $10 \%$ intervals. Hours below $65 \%$ and above $95 \%$ were also calculated. Leaf wetness at various temperature intervals was calculated using the same range and intervals as the temperature subvariables.

Field microclimate data from three seasons (1998 to 2000) were combined for logistic regression analysis. During these seasons, the plots were visually assessed twice weekly to determine if new lesions had appeared. Analyses of disease and weather data were conducted using the LOGISTIC procedure in SAS. The method of parameter estimation used was maximum likelihood estimation, which is most accurate with observations that are fully independent. Since our observations were not independent (observations within each year were repeated measurements from the same field over time), a second method of parameter estimation was also used. This approach, called generalized estimating equation methodology, allows the user to specify a cluster variable and a correlation matrix. Using the GENMOD procedure of SAS, an independent variable that identified the year/field was included and within each year a longitudinal auto-regressive dependence was specified.

Candidate models were identified using stepwise variable selection (significance level for entry $=0.05$ and significance level to stay $=0.05$ ) or score chi-square values (score selection option in SAS). While a number of different tests are available to evaluate the goodness-of-fit of a logistic regression model, most approaches make the assumption that for each combination of predictor variable values (each combination is a covariate pattern) there are at least five observations per outcome type. With our data, all of the predictor variables were continuous and therefore almost every observation had a unique covariate pattern. Under these conditions, Hosmer and Lemeshow (10) recommend a chi-square analysis of the expected frequency of observation types at various probability levels (based on the model) versus the observed frequencies. The result of this test, called the Hosmer and Lemehow goodness-of-fit test, was the primary criterion by which the models were evaluated.

Accuracy, sensitivity, and specificity of the models were also compared. Because logistic regression predicts a probability of an event occurrence that will always be between 0 and 1 , the dichotomous variable ("new infections" or "no new infections") was derived by defining a cut-point. When the estimated probability exceeded the cutpoint, the derived variable was set to 1 (last 10 days were conducive to infection), otherwise, it was set to 0 (last 10 days not conducive to infection). Receiver operating characteristic (ROC) curves were evaluated to select cut-point values for each model (17). A ROC curve illustrates, for a particular algorithm, the relationship between sensitivity (the proportion of conducive days which are correctly identified as such) and specificity (the proportion of nonconductive days correctly identified) at

Table 1. Analysis of the variation in percent leaf area diseased on inoculated, detached rose leaves incubated at varying temperatures $\left(5,10,15,20\right.$, and $\left.25^{\circ} \mathrm{C}\right)$ and with varying durations of leaf wetness $(2,4,6,8,10,12$, and $24 \mathrm{~h})$

\begin{tabular}{lrcc}
\hline Source of variation & df & Mean square & $\boldsymbol{P}>\boldsymbol{F}$ \\
\hline Replication & 5 & 54.4777 & $<0.0001$ \\
Temperature $(T)$ & 4 & 137.6635 & $<0.0001$ \\
Temperature $\times$ Replication $\left(\right.$ error $_{\mathrm{a}}$ ) & 20 & 4.2207 & $\ldots$ \\
Leaf Wetness Duration $(w)$ & 6 & 111.1849 & $<0.0001$ \\
$T \times w$ interaction & 24 & 9.7221 & $<0.0001$ \\
Error & 150 & 1.4506 & $\ldots$ \\
\hline
\end{tabular}

a number of different probability cut-points (17).

Preliminary validation of the microclimate model was conducted with a data set from 1998 and 1999 that had not been used in the development of the model. These data were collected by weather stations (Adcon Telemetry Inc.) placed at other locations in the rose production area. The test data were input into a given regression equation and the resulting probability was interpreted as either conducive or not conducive based on a selected cut-point value. In this way, the number of days classified correctly by the model was determined.

\section{RESULTS}

Experiments in controlled environments. The severity of infection, as measured by the percentage of the leaf area exhibiting symptoms, was significantly affected by temperature and leaf wetness duration $(P<0.0001$; Table 1$)$. The number of hours of leaf wetness required for infection of at least $7 \%$ of the leaf (rating $\geq 2$ ) varied with temperature. At 5, 10, 15, 20 , and $25^{\circ} \mathrm{C}$, this level of infection required $24,8,6,4$, and $4 \mathrm{~h}$ of leaf wetness, respectively (Fig. 1). The highest severity of infection (rating $\geq 6$ ) occurred at 10, 15, and $20^{\circ} \mathrm{C}$ with at least 24,8 , and $6 \mathrm{~h}$ of leaf wetness, respectively (Fig. 1). Although infection at $25^{\circ} \mathrm{C}$ required only $4 \mathrm{~h}$ of leaf wetness, overall severity of infection was lower, suggesting that fewer spores were capable of infecting at this temperature (Fig. 1).

Models of the infection (INF) response surface were obtained by two statistical methods. Because analysis of variance revealed a significant block (inoculation) effect (Table 1), individual data points were used for modeling rather than averages. Based on linear regression analysis of transformed data, two second-order polynomial models were selected, a nineparameter model with all possible interaction terms:

$\mathrm{INF}=1.24-0.42 T-1.09 w+0.27 T \times w+$ $0.015 T^{2}+0.041 w^{2}-0.0083 T \times w^{2}-$ $0.0078 T^{2} \times w+0.00023 T^{2} \times w^{2}$

and a five-parameter model without interaction terms:

$\mathrm{INF}=-7.98+0.97 T+0.74 w-0.027 T^{2}-$ $0.019 w^{2}$

All parameter estimates were significant (Table 2). The nine- and five-parameter models had $R^{2}$ values of 0.66 and 0.60 , respectively. The full model was an adequate fit to the data while the reduced model was not (lack-of-fit $P$ values equal to 0.0243 and $<0.0001$, respectively). For both models, regression of observed against predicted values produced regression equations with intercept and slope not significantly different from 0 and 1 , respectively (data not presented). The full polynomial model predicted that $17.9^{\circ} \mathrm{C}$ is 
the optimal temperature and $18 \mathrm{~h}$ is the optimal duration of wetness for infection.

Using nonlinear regression and by integration of the equations for the response to temperature with the equation for response to wetness duration we obtained the equation

$\mathrm{INF}=\left(10 /\left\{\exp \left[(T-16.72)^{2} \times 0.0076\right]\right\}\right) \times$ $\left\{1-\exp \left[-(0.106 w)^{1.255}\right]\right\}$

These parameter estimates and their associated statistics are presented in Table 3. This model had a pseudo- $R^{2}$ value of 0.58 and a significant lack-of-fit $(P<0.0001)$. Regression of observed against predicted values produced an equation with intercept and slope not significantly different from 0 and 1 , respectively (data not presented). This model predicted that the upper asymptote of the response to wetness was highest at $16.7^{\circ} \mathrm{C}$ (Fig 1.) and the optimal duration of wetness was $24 \mathrm{~h}$ (Fig. 1).

The latent period varied significantly with temperature $(P<0.001)$, and there was a significant isolate by temperature interaction ( $P=0.017$; Fig. 2$)$. No symptoms or sporulation occurred at $5^{\circ} \mathrm{C}$, although when boxes were removed from the growth chamber after 10 days and returned to room temperature, symptoms soon became visible, suggesting that colonization did occur at $5^{\circ} \mathrm{C}$ (data not presented). At $10^{\circ} \mathrm{C}$, sporulation began 7 or 8 days after inoculation, while at 15,20 , or $25^{\circ} \mathrm{C}$, sporulation began 4 or 5 days after inoculation (Fig. 2). The two isolates behaved similarly at $15^{\circ} \mathrm{C}$, but differently at 10,20 , and $25^{\circ} \mathrm{C}$. At these temperatures, isolate 99-1 began sporulating sooner than isolate $99-2$, but at $10^{\circ} \mathrm{C}$ isolate $99-1$ resulted in fewer sporulating leaves 10 days after inoculation than did isolate 99-2 (Fig. 2). In general, the pathogen began sporulating before leaf symptoms appeared when leaves were incubated at 10 or $15^{\circ} \mathrm{C}$, while symptoms preceded sporulation when leaves were incubated at 20 or $25^{\circ} \mathrm{C}$ (data not presented).

Models based on microclimate data. Each observation in the analysis consisted of 1 day, of which there were 226 in total (12 February to 27 April 1998, 20 February to 15 May 1999 , and 7 March to 11 May 2000). New downy mildew lesions appeared on every scouting date during two periods, 12 February to 27 April 1998 and 7 March to 3 April 2000. These days were categorized as conducive to downy mildew for logistic regression analysis. The dichotomous outcome variable was disease occurrence where "new infections" = 1 and "no new infections"= 0 . Fifty-seven microclimate variables were included in the analysis as predictor variables.

The best logistic regression model (model 1) contained three variables:(i) 10day cumulative number of hours of leaf wetness when temperatures were less than $20^{\circ} \mathrm{C}(x)$; (ii) the 10-day cumulative number of hours of temperatures between 15 and $20^{\circ} \mathrm{C}(y)$, (iii) and the 10-day cumulative number of hours of temperatures exceeding $30^{\circ} \mathrm{C}(z)$. In model 1 , the predicted probability of new infections appearing on a given day was given by

$\mathrm{PROB}=1 /[1+\exp (-23.84-0.45 x+1.53 y$ $+1.79 z)]$

Based on the criteria of Hosmer and Lemeshow (10), four observations were identified as outliers and removed. The Hosmer and Lemeshow goodness-of-fit test had a nonsignificant chi-square value of $0.02(\mathrm{df}=2, P=0.99)$, indicating a very
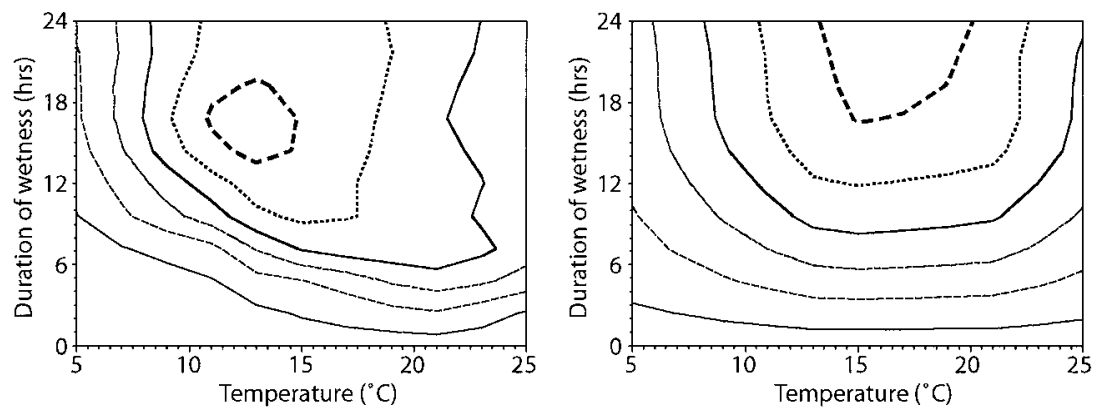

Fig. 1. Effect of temperature and duration of leaf wetness on infection. Observed values (left) are means of eight observations. Predicted values (right) are based on a nonlinear model. Rating scale of leaf area symptomatic is as follows: $0=0$ to $1.25 \% ; 1=1.25$ to $6.25 \% ; 2=6.25$ to $15.5 \% ; 3=15.5$ to $28 \% ; 4=28$ to $42.5 \% ; 5=42.5$ to $57.5 \% ; 6=57.5$ to $72 \% ; 7=72$ to $84.5 \% ; 8=84.5$ to $93.75 \%$; $9=93.75$ to $98.75 \%$; and $10=98.75$ to $100 \%$. Contour lines delineate leaf area ratings of approximately 1 (outermost) to 9 (innermost). Interpolation and contours calculated using SAS procedures G3GRID and GCONTOUR.

Table 2. Parameter estimates and associated statistics from two polynomial response surface models of the combined effects of temperature and duration of leaf wetness on downy mildew infection of inoculated, detached rose leaves incubated under controlled conditions

\begin{tabular}{lccc}
\hline Variable & Parameter estimate & Standard error & $\boldsymbol{t}$ value $^{\mathbf{a}}$ \\
\hline Nine-parameter model & & & \\
Intercept & 1.2427 & 1.8866 & 0.66 \\
Temperature $(T)$ & -0.4208 & 0.2876 & -1.46 \\
Leaf wetness duration $(w)$ & -1.093 & 0.3661 & $-2.99^{*}$ \\
$T^{2}$ & 0.0154 & 0.0094 & 1.63 \\
$w^{2}$ & 0.041 & 0.01328 & $3.09^{*}$ \\
$T \times w$ & 0.2651 & 0.05581 & $4.75^{*}$ \\
$T^{2} \times w^{2}$ & 0.0002 & 0.00007 & $3.52^{*}$ \\
$T \times w^{2}$ & -0.0083 & 0.002 & $-4.09^{*}$ \\
$T^{2} \times w$ & -0.0078 & 0.002 & $-4.28^{*}$ \\
Five-parameter model & & & \\
Intercept & -7.977 & 0.7752 & $-10.29^{*}$ \\
$T$ & 0.9689 & 0.1012 & $9.57^{*}$ \\
$w$ & 0.7378 & 0.0822 & $8.98^{*}$ \\
$T^{2}$ & -0.0269 & 0.0033 & $-8.13^{*}$ \\
$w^{2}$ & -0.0189 & 0.003 & $-6.33^{*}$ \\
\hline
\end{tabular}

a * indicates $t$ value significant at $P<0.05$.

Table 3. Parameter estimates and associated statistics for the model INF $=\left(10 /\left\{\exp \left[(T-F)^{2} G\right]\right\}\right) \times\{1$ $\left.-\exp \left[-(B w)^{\mathrm{D}}\right]\right\}$ that described the response of relative infection (INF) to the effects of temperature $(T)$ and duration of leaf wetness $(w)$

\begin{tabular}{lcccc}
\hline & & & \multicolumn{2}{c}{ Asymptotic 95\% confidence interval } \\
\cline { 5 - 5 } Parameter $^{\mathbf{a}}$ & Estimate & Asymptotic standard error & Lower & Upper \\
\hline$F$ & 16.718 & 0.6658 & 15.3541 & 18.0819 \\
$G$ & 0.00757 & 0.00122 & 0.00506 & 0.0101 \\
$B$ & 0.1056 & 0.00584 & 0.0941 & 0.1171 \\
$D$ & 1.2549 & 0.1486 & 0.9619 & 1.5479 \\
\hline
\end{tabular}

a Parameter estimates were obtained in two steps, first using the equation $\mathrm{INF}_{\max }=\mathrm{A}=10 /\{\exp [(T-$ $\left.\left.F)^{2} G\right]\right\}$ and incorporating these results and analyzing the equation $\mathrm{INF}=A \times\left\{1-\exp \left[-(B w)^{\mathrm{D}}\right]\right\}$. 
number of hours of leaf wetness $(x)$. After the elimination of three outliers in the data set, the Hosmer and Lemeshow goodnessof-fit test had a chi-square value of 12.73 ( $\mathrm{df}=8, P=0.12$ ), indicating a satisfactory fit of the data to the model. Both the intercept and the regression coefficient were significant $(P=0.05$; Table 4$)$. Model 2 predicted

$\mathrm{PROB}=1 /[1+\exp (7.76-0.09 x)]$

There was a critical value for cumulative hours of leaf wetness around $84.4 \mathrm{~h}$ or 8.4 $\mathrm{h}$ per night. When the duration of wetness exceeded this level, the probability of new infections appearing surpassed 0.5 .

Model based on mesoclimate data. The analysis of mesoclimate data included 312
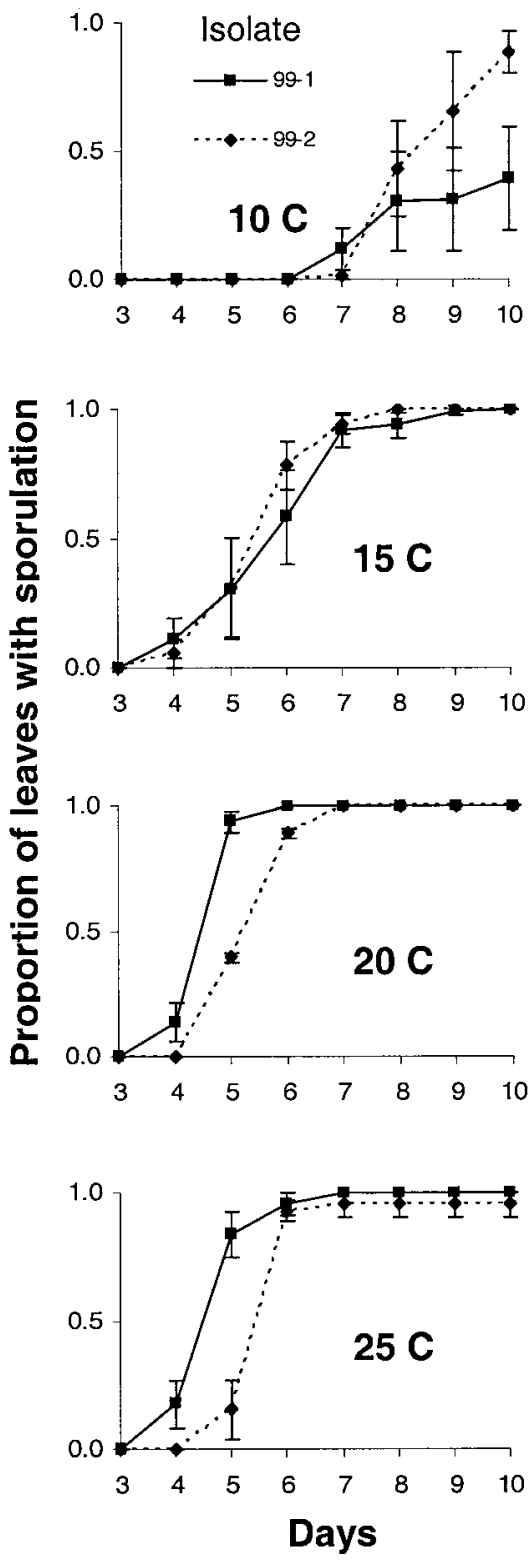

Fig. 2. Observed effect of constant temperature on the latent period (time from infection to sporulation) of Peronospora sparsa colonizing rose. Each point is the mean of six (isolate 991) or three (isolate 99-2) observations. Bars represent the standard error of the mean. observations (1 February to 15 May of each year) and 19 predictor variables. The same dates were categorized as conducive as described for the microclimate analysis above.

The model selected by stepwise logistic regression of data from the regional weather station (model 3) had four variables: (i) the cumulative number of hours when RH exceeded $75 \%$ in the last 10 days (w); (ii) the cumulative number of hours when temperatures were 10 to $15^{\circ} \mathrm{C}$ in the last 10 days $(x)$; (iii) the cumulative number of hours when temperatures were 15 to $20^{\circ} \mathrm{C}$ in the last 10 days (y); and (iv) the cumulative number of hours when temperatures were 20 to $25^{\circ} \mathrm{C}$ in the last 10 days (z). Model 3 predicted

PROB $=1 /[1+\exp (17.94-0.11 w+0.06 x$ $-0.07 y-0.17 z)]$

Eleven observations (of the original 312) were identified as outliers and were removed. The Hosmer and Lemeshow goodness-of-fit test had a chi-square value of 12.97 (df $=8, P=0.11$ ), indicating a satisfactory fit. All of the estimated parameters were statistically different from zero $(P=$ 0.05 ; Table 4 ). For all models, the generalized estimating equation methodology (GENMOD procedure) produced very similar parameter estimates to the LOGISTIC procedure (data not presented).
Model comparison. When the cut-point for the best three-variable microclimate model was set at 0.42 , the model correctly identified 102 days with new infections, and correctly identified 117 days as lacking new infections (Table 5). On 3 days, the model predicted new infections when in fact none appeared (false positive rate $=$ 0.025). The false negative rate was 0 , as there were no days when the model would have predicted a lack of new infections when, in fact, new infections did appear. The second three-variable model was less accurate (data not presented).

When the cut-point for the singlevariable model was set to 0.09 , the model correctly identified 102 days with new infections and 86 days as lacking new infections (Table 5). However, it falsely identified 35 days as having infections (false positive rate $=0.29$ ) and 0 days as not having infections (false negative rate $=0$ ).

In the mesoclimate model, there was no cut-point value at which false negatives could be avoided without increasing the false positive rate to over $50 \%$ (i.e., over $50 \%$ of days declared conducive would actually be nonconductive; Table 5).

Preliminary validation. To conduct a preliminary validation of the two microclimate models, the predicted probabilities were calculated from their respective re-

Table 4. Parameter estimates and associated statistics of logistic regression parameters from three separate models of the probability of rose downy mildew occurrence based on data from in-field (microclimate) or regional (mesoclimate) weather stations

\begin{tabular}{lcccc}
\hline Variable & $\begin{array}{c}\text { Parameter } \\
\text { estimate }\end{array}$ & Standard error & $\begin{array}{c}\text { Wald } \\
\text { Chi-square } \boldsymbol{P}\end{array}$ & $\begin{array}{c}\text { Standardized } \\
\text { estimate }\end{array}$ \\
\hline Model 1 & & & & \\
Intercept & 23.8428 & 11.6951 & 0.0415 & 11.5715 \\
$x$ & 0.4507 & 0.2200 & 0.0405 & -9.1046 \\
$y$ & -1.5307 & 0.7411 & 0.0389 & -12.6394 \\
$z$ & -1.7878 & 0.8830 & 0.0429 & \\
Model 2 & & & & 2.352 \\
Intercept & -7.7632 & 1.0203 & $<0.0001$ & \\
$x$ & 0.0919 & 0.0126 & $<0.0001$ & -0.5143 \\
Model 3 & & & & 0.6566 \\
Intercept & -17.9375 & 2.4958 & $<0.0001$ & 1.7811 \\
$w$ & -0.0560 & 0.0135 & $<0.0001$ & 2.5371 \\
$x$ & 0.0674 & 0.0151 & $<0.0001$ & $<0.0001$ \\
$y$
\end{tabular}

Table 5. Accuracy, sensitivity, and specificity of three logistic regression models developed to predict the effect of environmental parameters on occurrence of rose downy mildew in commercial fields. Model 1, based on 10-day cumulative microclimate data, includes the duration of leaf wetness (when $T<20^{\circ} \mathrm{C}$ ), the duration of temperatures 15 to $20^{\circ} \mathrm{C}$, and the duration of temperatures $>30^{\circ} \mathrm{C}$. Model 2, also based on 10-day cumulative microclimate data, includes the duration of leaf wetness. Model 3, based on 10-day cumulative mesoclimate data, includes the duration of relative humidity $>75 \%$, the duration of temperatures 10 to $15^{\circ} \mathrm{C}$, the duration of temperatures 15 to $20^{\circ} \mathrm{C}$, and the duration of temperatures 20 to $25^{\circ} \mathrm{C}$

\begin{tabular}{|c|c|c|c|c|c|c|}
\hline \multirow[b]{2}{*}{ Model } & \multicolumn{3}{|c|}{ Development } & \multicolumn{3}{|c|}{ Validation } \\
\hline & Accuracy $^{\mathbf{a}}$ & Sensitivity $^{b}$ & Specificity $^{c}$ & Accuracy $^{\mathbf{a}}$ & Sensitivity $^{b}$ & Specificity $^{c}$ \\
\hline 1 & 98.6 & 100 & 97.5 & 79.1 & 77 & 80.5 \\
\hline 2 & 84.7 & 100 & 71.1 & 76.4 & 85.2 & 70.1 \\
\hline 3 & 72.1 & 89.9 & 63.4 & $\ldots$ & $\ldots$ & $\ldots$ \\
\hline
\end{tabular}

a Accuracy = percentage of days classified correctly.

b Sensitivity = percentage of days with new downy mildew infections classified correctly.

${ }^{c}$ Specificity $=$ percentage of days without new downy mildew infections classified correctly. 
gression equations using weather data from two field locations that were not used in model development. Using 63 observations from 1998 and 85 observations from 1999, the three-variable model (with the cutpoint set to 0.42 ) correctly identified 47 conducive days and 70 nonconducive days (Table 5). However, it incorrectly classified 17 days as conducive when, in fact, no infections had been observed (false positive rate $=0.2$ ), and it incorrectly classified 14 days as nonconducive when, in fact, infections had been observed (false negative rate $=0.23$ ). The single variable microclimate model (with the cut-point set to 0.50 ) had a false positive rate of 0.3 and a false negative rate of 0.15 .

\section{DISCUSSION}

This is the first report of the effects of temperature, leaf wetness, and relative humidity on the infection and colonization of rose by Peronospora sparsa. Results from controlled experiments in this study confirmed observations by Baker (1) that as little as $4 \mathrm{~h}$ leaf wetness was sufficient for infection of roses by $P$. sparsa when the temperature is optimal. However, temperatures at night in the San Joaquin Valley in March and April $\left(\sim 10^{\circ} \mathrm{C}\right)$ are less than optimal for infection. At temperatures near $10^{\circ} \mathrm{C}$, our experiments indicated that at least $8 \mathrm{~h}$ leaf wetness were required for significant levels of infection. Regression analysis of data from field epidemics confirmed these results from the controlled experiments. Logistic regression identified critical leaf wetness duration of $84 \mathrm{~h}$ (accumulated over 10 days) that discriminated between periods that were conducive or nonconducive to downy mildew development.

Breese et al. (3) evaluated the effects of temperature on in vitro sporangium germination of four isolates of $P$. sparsa, two from rose and two from tummelberry (a Rubus sp. hybrid). Maximum in vitro sporangium germination occurred from a low of 2 to $8^{\circ} \mathrm{C}$ to a high of 14 to $18^{\circ} \mathrm{C}$, depending on the isolate (3). Although no statistics were presented, the different isolates, which came from different geographic locations, seemed to differ significantly in their response to temperature. However, all isolates failed to germinate at temperatures above $26^{\circ} \mathrm{C}$. On tummelberry leaf disks, infection by a Rubus sp. isolate was highest at $15^{\circ} \mathrm{C}$ and was reduced at temperatures above $23^{\circ} \mathrm{C}$. In these studies, infection of tummelberry leaves was evaluated simply on a presence/absence basis, without considering severity of infection. No infection experiments were conducted with a rose isolate or on rose leaves. In the only other published work, Baker (1) found that sporangia of P. sparsa did not germinate at $4.5^{\circ} \mathrm{C}$, but did survive exposure to such temperatures. The optimum temperature for germination was $18.3^{\circ} \mathrm{C}$, with a slight decrease at $21^{\circ} \mathrm{C}$ and no germination at $26.6^{\circ} \mathrm{C}$ (1). When temperatures of $26.6^{\circ} \mathrm{C}$ were maintained for 24 $\mathrm{h}$, the spores were killed. However, the experimental methods were not described and no statistics were presented.

In our study, infection of rose leaves by $P$. sparsa occurred over a broad range of temperatures from 5 to $25^{\circ} \mathrm{C}$. Our observations at low temperatures were similar to those of Breese et al. (3), in which germination in vitro occurred at temperatures as low as $2^{\circ} \mathrm{C}$. On the other hand, Breese et al. (3) saw only very low levels of spore germination and leaf infection at $25^{\circ} \mathrm{C}$, whereas $25^{\circ} \mathrm{C}$ was only moderately inhibitory to infection by California isolates, suggesting possible adaptation to warmer temperatures. In our study, optimum infection occurred between 15 and $20^{\circ} \mathrm{C}$ while Breese et al. (3) found $14.7^{\circ} \mathrm{C}$ to be optimal for infection of tummelberry (Rubus sp.), with a significant decrease at 18 or $21^{\circ} \mathrm{C}$. Variation in temperature ranges for germination of isolates with different geographical origins has been observed in the downy mildew pathogen Sclerospora sorghi (2). Studies using a larger collection of $P$. sparsa isolates are needed to investigate the possibility of geographic adaptation. For all three response surface models, the distribution of residuals appeared random with respect to wetness duration, but exhibited a slight curvilinear trend with respect to temperature (data not presented). This indicates that the modeling of the response to temperature could be improved.

In some of the replicate inoculations, extended leaf wetness periods $(24 \mathrm{~h})$ at optimal temperatures resulted in relative infection values that were lower than the maximum observed for that replicate. Although the effect was slight, it suggests that long wetness durations may sometimes inhibit the infection or colonization process. The polynomial model exaggerates this phenomenon, while the nonlinear model underestimates it by overestimating infection levels at optimal temperatures and long wetness durations. Another problem with the polynomial models is their prediction of negative response values, which are not reasonable. Improvement of both types of models could be made with further studies and adjustment of parameter estimates.

The latent period of $P$. sparsa on roses was very short. The optimum temperature for colonization was 20 or $25^{\circ} \mathrm{C}$. At these temperatures, significant proportions of leaves were symptomatic 4 days after inoculation and were sporulating 5 days after inoculation. The average daily temperature in Kern County in March and April is typically between 10 and $20^{\circ} \mathrm{C}$. At these temperatures, symptoms would appear and sporulation would begin in only 4 to 7 days. This is a very rapid cycle, not uncommon for a downy mildew, which may explain the difficulty growers have experi- enced in control of the disease with protective fungicides applied on a 14-day interval.

A regression model of field epidemics was developed that was remarkably well fit to the development data. The ROC curve for the three-variable microclimate model in this study had the ideal shape, with the curve "pushed" into the upper left-hand corner. This indicates a model with high sensitivity and high specificity. In comparison, the single variable model had somewhat lower predictive power. Given the high error rate of the mesoclimate model, it probably would not be useful as part of a disease-warning system. The failure of the mesoclimate model is likely due to the fact that leaf wetness data are not available from the CIMIS stations. Leaf wetness duration is apparently a critical factor based on its relative importance in our microclimate models, the results from our controlled experiments, and studies of other downy mildews $(6,13-15)$. In addition, the relative humidity measurements made at the CIMIS station are likely only weakly correlated with the humidity levels found within a rose canopy.

The development data were less than ideal as they were collected during one season with no disease at all (1999) and one season with very severe disease (1998). The weather in those seasons was also extreme, as there was a severe El Niño event during 1997 to 1998 and a moderate La Niña event during 1998 to 1999 . Continued validation of these models should involve data from other years and subsequent adjustment of the regression parameters and probability cut-point values. The probability cut-point or threshold selected for use with a particular model should be adjusted based on the risk attitudes of the user and their tolerance for different types of errors (false positives versus false negatives) (17).

This is the first attempt to model the response of rose downy mildew to environmental variables. The data used in the preliminary validation of the model were a poorer fit to the model than the development data. This is to be expected, however, since it is always the case that a model will perform optimally with the development data. Empirical improvement of the model with data from future seasons should result in a model that could be used as part of a disease-warning system.

\section{LITERATURE CITED}

1. Baker, K. F. 1953. Recent epidemics of downy mildew of rose. Plant Dis. Rep. 37:331-339.

2. Bonde, M. R., Schmitt, C. G., and Dapper, R. W. 1978. Effects of dew-period temperature on germination of conidia and systemic infection of maize by Sclerospora sorghi. Phytopathology 68:219-222.

3. Breese, W. A., Shattock, R. C., Williamson, B., and Hackett, C. 1994. In vitro spore germination and infection of cultivars of Rubus and Rosa by downy mildews from both hosts. Ann. Appl. Biol. 125:73-85. 
4. Campbell, C. L., and Madden, L. V. 1990. Introduction to Plant Disease Epidemiology. John Wiley and Sons, New York.

5. Carisse, O., Bourgeois, G., and Duthie, J. A. 2000. Influence of temperature and leaf wetness duration on infection of strawberry leaves by Mycosphaerella fragariae. Phytopathology 90:1120-1125.

6. Cohen, Y. 1977. The combined effects of temperature, leaf wetness, and inoculum concentration on infection of cucumbers with Pseudoperonospora cubensis. Can. J. Bot. 55:1478-1487.

7. de Visser, C. L. M. 1998. Development of a downy mildew advisory model based on downcast. Eur. J. Plant Pathol. 104:933-943.

8. Dhingra, O. D., and Sinclair, J. B. 1995. Basic Plant Pathology Methods. CRC Press, Inc.,
Boca Raton, FL.

9. Duthie, J. A. 1997. Models of the response of foliar parasites to the combined effects of temperature and duration of wetness. Phytopathology 87:1088-1095.

10. Hosmer, D. W., and Lemeshow, S. 2000. Applied logistic regression. John Wiley and Sons, New York.

11. Kern County Agricultural Commissioners Office. 2000. Kern County 2000 Crop Report.

12. Madden, L. V., Ellis, M. A., Lalancette, N., Hughes, G., and Wilson, L. L. 2000. Evaluation of a disease warning system for downy mildew of grapes. Plant Dis. 84:549-554.

13. Populer, C. 1981. Epidemiology of downy mildews. Pages 57-105 in: The Downy Mildews. D. M. Spencer, ed. Academic Press, New York.
14. Scherm, H., and van Bruggen, A. H. C. 1993. Response surface models for germination and infection of Bremia lactucae, the fungus causing downy mildew of lettuce. Ecol. Model. 65:281-296.

15. Scherm, H., and van Bruggen, A. H. C. 1995 Concurrent spore release and infection of lettuce by Bremia lactucae during mornings with prolonged leaf wetness. Phytopathology 85:552-555.

16. Wu, B. M., Subbarao, K. V., van Bruggen, A. H. C., and Koike, S. T. 2001. Comparison of three fungicide spray advisories for lettuce downy mildew. Plant Dis. 85:895-900.

17. Yuen, J., Twengstöm, E., and Sigvald, R. 1996. Calibration and verification of risk algorithms using logistic regression. Eur. J. Plant Pathol. 102:847-854. 